\title{
Frontal white matter integrity as an endophenotype for schizophrenia: diffusion tensor imaging in monozygotic twins and patients' nonpsychotic relatives
}

\author{
Jazmin Camchong ${ }^{1,2 *}$, Kelvin O. Lim ${ }^{1,3}$, Scott R. Sponheim ${ }^{3,2,1}$ and Angus W. MacDonald III 1,2 \\ 1 Department of Psychiatry, University of Minnesota, Minneapolis, MN, USA \\ 2 Department of Psychology, University of Minnesota, Minneapolis, MN, USA \\ ${ }^{3}$ Veterans Affairs Medical Center, Minneapolis, MN, USA
}

Edited by:

Kenneth Hugdahl, University of Bergen, Norway

Reviewed by:

Ingrid Agartz, University of Oslo, Norway

Kenneth Hugdahl, University of

Bergen, Norway

*Correspondence:

Jazmin Camchong, Departments of

Psychiatry and Psychology, University

of Minnesota, 75 East River Road,

Minneapolis, MN 55455, USA.

e-mail: camch001@umn.edu
Diffusion tensor imaging (DTI) provides anatomical connectivity information by examining the directional organization of white matter microstructure. Anatomical connectivity and its abnormalities may be heritable traits associated with schizophrenia. To further examine this hypothesis, two studies were conducted to compare anatomical connectivity between (a) monozygotic (MZ) twin pairs and random pairings among twins and (b) first-degree relatives of schizophrenia patients and a healthy control group. Analyses focused on frontal regions of the brain following previous findings of anatomical connectivity abnormalities associated with schizophrenia. For Study 1, eighteen MZ twin pairs (11 female pairs, age: $M=25.44, S D=5.69$ ) were recruited. For Study 2, twenty-two first-degree relatives of schizophrenia patients (14 females, age: $M=48.50, S D=8.22$ ), and 30 healthy controls (12 females, age: $M=43.83$, $S D=11.39$ ) were recruited. Fractional anisotropy (FA), a white matter directional organization metric, was measured with DTI. In Study 1, FA values were more strongly correlated between $\mathrm{MZ}$ twin pairs than between randomly generated pairs in genu of corpus callosum, anterior cingulum and forceps minor. In Study 2, relatives of schizophrenia patients showed reduced FA values in medial frontal white matter $(p<0.05$, corrected). The present study suggested that anatomical connectivity in medial prefrontal cortex appeared significantly heritable within $\mathrm{MZ}$ twin pairs, an important criterion in the development of an endophenotype. In addition, altered medial frontal white matter integrity found in non-affected relatives of schizophrenia patients seems to suggest that reduced white matter integrity in medial frontal regions of the brain might be associated with the genetic liability to schizophrenia.

Keywords: diffusion tensor imaging , fractional anisotropy, monozygotic twin pairs, schizophrenia relatives, endophenotype, white matter, medial frontal

\section{INTRODUCTION}

Quality of brain functioning is dependent upon the proper interaction of multiple brain regions and neural networks. These interactions occur on multiple spatial and temporal scales, i.e. fast and slow communication between circumscribed and distributed brain areas. Disruption of the normal interactions between brain regions may be related to cognitive deficits and clinical symptoms found in psychiatric populations (Hoptman et al., 2004; Fujiwara et al., 2007; Manoach et al., 2007; Skelly et al., 2008; Takei et al., 2008; Camchong et al., 2009; Whitfield-Gabrieli et al., 2009).

Successful communication between brain areas depends on the underlying structure of neural networks (e.g. axonal and dendritic networks connecting brain areas). Diffusion tensor imaging (DTI) is an in vivo approach that has been used to look at interaction of brain regions by examining the directional organization of white matter microstructure. DTI provides information about the magnitude and direction of water diffusion within white matter tissue (Basser and Pierpaoli, 1996). Water diffusion in white matter is restricted by myelin, a lipid- and protein-rich axonal covering. Intracellular water within myelinated axons diffuses in a more directional manner compared to diffusion in unmyelinated axons. The directional properties of water diffusion can be measured with fractional anisotropy (FA), a commonly derived scalar measure of DTI. High FA values correspond to preferential diffusion along one direction, indicating a high level of tissue organization (Basser and Pierpaoli, 1996).

DTI studies examining schizophrenia have found lower levels of tissue organization, manifested as lower FA values in schizophrenia patients (Kubicki et al., 2003., 2005, 2008; Sun et al., 2003; Hoptman et al., 2004; Kanaan et al., 2005; Fujiwara et al., 2007; Manoach et al., 2007; Schlosser et al., 2007; Cheung et al., 2008; Skelly et al., 2008; Takei et al., 2008; Camchong et al., 2009; Ellison-Wright and Bullmore, 2009) and in individuals at high risk for developing the disease (Hoptman et al., 2008; Munoz Maniega et al., 2008; Karlsgodt et al., 2009) when compared to controls. Reduced white matter connectivity has been found in a number of regions in schizophrenia patients including fornix, genu and splenium of corpus callosum, anterior cingulum bundle, superior occipito-frontal fasciculus, internal capsule and arcuate fasciculus (Kubicki et al., 2003, 2005, 2008; Sun et al., 2003; Fujiwara et al., 2007; Cheung et al., 2008).

Based on findings from a recent study conducted by our group (Camchong et al., 2009), this current study focused only on connectivity abnormalities in medial frontal regions of the brain (genu 
of corpus callosum, anterior cingulum bundle). Medial frontal abnormalities have been consistently found in both white matter and adjacent gray matter of schizophrenia patients (Sun et al., 2003; Fujiwara et al., 2007; Kubicki et al., 2008; Kyriakopoulos et al., 2008a; Camchong et al., 2009; Fitzsimmons et al., 2009). The investigation of altered medial prefrontal cortex found in schizophrenia patients is important because previous studies have found significant associations between severity of behavioral abnormalities (such as severity of psychopathology and severity of cognitive dysfunction) and altered medial prefrontal cortex connectivity (Hoptman et al., 2004; Manoach et al., 2007; Skelly et al., 2008; Takei et al., 2008; Camchong et al., 2009). In the current study, we were particularly interested in examining the possibility of considering altered anatomical connectivity in medial prefrontal cortex a heritable biological marker, or endophenotype, for schizophrenia.

An endophenotype is a measurable trait intermediate between the clinical manifestation of the disorder and its underlying genes (Gottesman and Gould, 2003). The endophenotype, then, is putatively a less complex correlate of the disorder than are clinical symptoms, is closer to gene action, and can aid in discovering the disorder's genetic etiology. White matter connectivity abnormalities in schizophrenia patients, indeed, meet the first criterion (Gottesman and Gould, 2003) proposed to be necessary for status as an endophenotype: that the trait is associated with the illness in the population (Sun et al., 2003; Fujiwara et al., 2007; Kubicki et al., 2008; Camchong et al., 2009). In the current study we will focus on two additional criteria necessary for endophenotype status (Gottesman and Gould, 2003): that the trait is heritable and that the endophenotype is found in non-affected family members at a higher rate than in the general population.

In order to investigate whether medial frontal white matter abnormalities are heritable, the correlation between healthy family members may be examined. Family members that share the highest degree of genetic makeup are monozygotic (MZ) twins. By examining the degree of similarities or correlation of white matter integrity between MZ twins we can explore the degree of heritability of this trait. To our knowledge, there are no studies that have examined heritability of white matter integrity in twin pairs.

Endophenotype status also requires that non-affected family members of schizophrenia patients show medial frontal white matter abnormalities. Findings of connectivity abnormalities in patients' relatives, however, are not very consistent; although frontal regions are usually implicated (Hoptman et al., 2008). A study by Hoptman et al. (2008) found that relatives of schizophrenia patients had reduced FA in white matter adjacent to inferior frontal gyrus, posterior cingulate and angular gyri. Munoz Maniega et al. (2008), focusing on four discrete regions of interest, found reduced FA in anterior limb of the internal capsule in relatives of schizophrenia patients, a white matter tract that has connections between the thalamus and frontal lobe. Karlsgodt et al. (2009), who also focused on specific regions of interest, found reduced FA in superior longitudinal fasciculus, a fronto-parietal connection, of relatives of schizophrenia patients. Taken together, these findings suggest that altered frontal white matter connectivity indeed shows promise as an endophenotype for schizophrenia. Medial frontal regions, in particular, may be an important area on which to focus, as connectivity deficits have been consistently found here (Sun et al.,
2003; Fujiwara et al., 2007; Kubicki et al., 2008; Kyriakopoulos et al., 2008b; Camchong et al., 2009), and this region has been shown to be involved in certain clinical and cognitive deficiencies present in schizophrenia patients (Manoach et al., 2007; Camchong et al., 2009; Whitfield-Gabrieli et al., 2009). Based on this literature, the present study focused the examination of white matter integrity on this region as a confirmatory analysis. An exploratory analysis was also conducted looking for group differences in the whole brain.

In order to further explore the hypothesis that white matter disorganization in medial frontal regions is a schizophrenia-related endophenotype, we conducted two studies. Study 1 examined the degree of correlation (i.e. heritability) of white matter connectivity, as indexed by DTI-derived FA values, in medial frontal regions of healthy MZ twins. Heritability is arguably one of the most important criteria to be met by a potential endophenotype (Gottesman and Gould, 2003). Given that MZ twins share nearly identical genetic loadings for the presence of any particular physical trait, the degree of similarity between members of a twin pair reveals the importance of genes for that trait, assuming no assertive mating among the twins' parents for traits associated with FA values, and a minimal contribution of shared environmental factors. With regard to white matter organization, therefore, we hypothesized that $\mathrm{MZ}$ twins would have a stronger degree of correlation than in pairs of non-related individuals (i.e. white matter connectivity would be heritable). Study 2 compared white matter connectivity deficits between first-degree biological relatives of schizophrenia patients and a control group. We hypothesized that the relatives would show similar anatomical connectivity abnormalities as the patients when compared to the control group; these abnormalities, however, would be found to a lesser degree in relatives than what has been previously found in schizophrenia patients.

\section{MATERIALS AND METHODS STUDY 1 \\ Participants}

Eighteen MZ twin pairs (11 female pairs, age: $M=25.44, \mathrm{SD}=5.69$ ) were recruited from a twin registry from the University of Minnesota. All participants were free of neurological problems. Exclusion factors were DSM-IV criteria for Alcohol or Substance Abuse or Dependence within 3 months prior to scanning; significant medical illness; or head injury resulting in loss of consciousness exceeding $30 \mathrm{~min}$.

\section{DTI data acquisition and analysis}

All subjects were scanned using a research-dedicated Siemens Trio 3 Tesla scanner (Erlangen, Germany) located at the Center for Magnetic Resonance Research at the University of Minnesota. A high-resolution T1-weighted anatomical image was acquired using a magnetization prepared rapid gradient echo sequence. DTI data were acquired axially using a dual spin echo, single shot, pulsed gradient, echo planar imagine (EPI) sequence $(\mathrm{TR}=6.2 \mathrm{~s}, \mathrm{TE}=85 \mathrm{~ms}$, 55 slices, voxel size $=2.5 \mathrm{~mm} \times 2.5 \mathrm{~mm} \times 2.5 \mathrm{~mm}, 0 \mathrm{~mm}$ skip, FOV $=240 \mathrm{~mm}, b$ value $=1000 \mathrm{~s} / \mathrm{mm} 2$ ). Diffusion was measured along 30 directions.

Data were preprocessed with FDT (FMRIB's Diffusion Toolbox) (Behrens et al., 2003). Data was corrected for effects of head movement and eddy currents by using affine registration to a reference 
volume (Reese et al., 2003). Voxel-wise statistical analysis of the FA data was carried out using TBSS (Tract-Based Spatial Statistics) (Smith et al., 2006), part of FSL (Smith et al., 2004). First, raw DTI data was brain-extracted using BET, and then FA images were created by fitting a tensor model to the raw diffusion data using FDT. All subjects' FA data were then aligned into a common space (Montreal Neurological Institute-152 brain) (Mazziotta et al., 1995) using the nonlinear registration IRTK (Rueckert et al., 1999). Next, the mean FA image was created and thinned to create a mean FA skeleton which represents the centers of all tracts common to the group. Each subject's aligned FA data was then projected onto this skeleton and the resulting data fed into voxel-wise cross-subject statistics.

In order to examine medial frontal similarities between $\mathrm{MZ}$ twins, a region of interest (ROI) was defined that included cingulum bundle and genu of corpus callosum. The ROI was created by overlaying the TBSS-generated skeleton with the John Hopkins University (JHU) DTI-based probabilistic atlas. To correct for multiple comparisons, a randomization test on correlation coefficients was used $^{1}$ (Howell, 2001, 2007) to generate a sampling distribution to test the significance of 15 voxels that showed the highest crosstwin correlations within the mask. In this analysis, twin 1 FA values were held constant, while twin 2 FA values were permuted 100,000 times in order to build a sampling distribution of $r$. The randomization program by Howell (Howell, 2001) provided a sample distribution of $r$ with 100,000 cases for each voxel, thereby providing a probability that the obtained $r$ for the original comparison is due by chance (uncorrected $p$-values).

\section{STUDY 2}

\section{Participants}

Twenty-two first-degree relatives of schizophrenia patients (14 females, age: $M=48.50, \mathrm{SD}=8.22$ ), and 30 healthy controls (12 females, age: $M=43.83, \mathrm{SD}=11.39$ ) were recruited. Three of the first-degree relatives of schizophrenia patients were parents, the remaining nineteen relatives were siblings. These participants were recruited from a larger, broader family study conducted through the Minneapolis VA Medical Center if they fulfilled the criteria outlined below. Participants were excluded if English was a second language, had an IQ less than 70 or a diagnosis of mental retardation, current alcohol or drug abuse, current drug dependence, a current or past central nervous system disease or condition, a medical condition or disease with likely significant central nervous system effects, history of head injury with skull fracture or loss of consciousness of greater than $30 \mathrm{~min}$, a physical problem that would render study measures difficult or impossible, a history of electroconvulsive therapy, and an age less than 18 or greater than 60 . Participants were additionally excluded for a current major depressive episode, current or previous use of anti-psychotic medications, a personal history of psychosis or bipolar affective disorder, or an Axis II Cluster A personality disorder. Lastly, controls were further excluded for a family history of psychosis or bipolar affective disorder. The Veteran's Affairs Medical Center and University of Minnesota Institution Review Boards approved the protocol.

${ }^{1}$ http://www.uvm.edu/ dhowell/StatPages/Resampling/RandomCorr/ randomization_Correlation.html

\section{DTI acquisition and analysis}

Data acquisition parameters were the same as in Study 1 for the first 12 relatives of schizophrenia patients and the first 16 healthy controls. For the remaining 10 relatives and 14 healthy controls DTI data were acquired axially using a dual spin echo, single shot, pulsed gradient, EPI sequence $(\mathrm{TR}=7.0 \mathrm{~s}, \mathrm{TE}=87 \mathrm{~ms}, 55$ slices, voxel size $=2.5 \mathrm{~mm} \times 2.5 \mathrm{~mm} \times 2.5 \mathrm{~mm}, 0 \mathrm{~mm}$ skip, FOV $=325 \mathrm{~mm}$, $b$ value $=1000 \mathrm{~s} / \mathrm{mm} 2$ ). Diffusion was measured along 30 directions. Data preprocessing was the same as in Study 1.

Voxel-wise $t$-test within the ROI (same as in Study 1) was conducted to examine regional group differences with Randomise from FSL51. Randomise ${ }^{2}$ is a permutation program that corrects for multiple comparisons by using the null distribution of the max (across the image) cluster size (Nichols and Holmes, 2002). Randomise enables modeling and inference using a cluster-based thresholding (critical $t$-value of 1.697). Effects of scanner and effects of age were added to the model as nuisance variables. Whole brain voxel-wise $t$-test was conducted as an exploratory analysis to look for group differences with Randomise.

The program tbss_fill ${ }^{3}$ from FSL was used for illustration purposes of final results for both studies. Tbss_fill thickens the thresholded stats image, filling it out into the local "tracts" seen in mean_FA (See Figure 1).

\section{RESULTS}

\section{STUDY 1}

Voxels found to be correlated between $\mathrm{MZ}$ twin pairs included genu of corpus callosum, anterior cingulum and white matter adjacent to medial frontal cortex (see Figure 1 and Table 1). FA values were more strongly correlated between $\mathrm{MZ}$ twin pairs than between randomly generated pairs in these regions (mean of obtained $r=0.58$; mean of $p$ value $=0.02$ ) .

\section{STUDY 2}

Mean ages in relatives $(M=48.50, \mathrm{SD}=8.22)$ were not significantly different from controls $(M=43.83, \mathrm{SD}=11.39)[t(2,50)=1.633$, $p=0.11]$. Between-groups $t$-test revealed significant differences in two discrete clusters in right genu of corpus callosum (See Figure 1 and Table 2). Relatives of schizophrenia patients had significantly lower FA values than controls in this region $(p<0.05$, corrected, $t$-values were greater or equal to 1.697). There were no significant effects of scanner covariate or age covariate and white matter FA. There were no group differences as a result of whole brain voxelwise $t$-test analysis.

\section{DISCUSSION}

The present study investigated the degree to which white matter disorganization in medial frontal regions is a biological marker or endophenotype associated with schizophrenia. White matter connectivity in medial frontal regions was examined (1) in healthy $\mathrm{MZ}$ twins in order to address heritability of white matter connectivity in this region and (2) in first-degree biological relatives of schizophrenia patients to investigate whether individuals at risk for developing the disease have similar alterations in frontal white matter

\footnotetext{
${ }^{2}$ http://www.fmrib.ox.ac.uk/fsl/randomise/index.html
}

${ }^{3}$ http://www.fmrib.ox.ac.uk/fsl/tbss/index.html\#display 


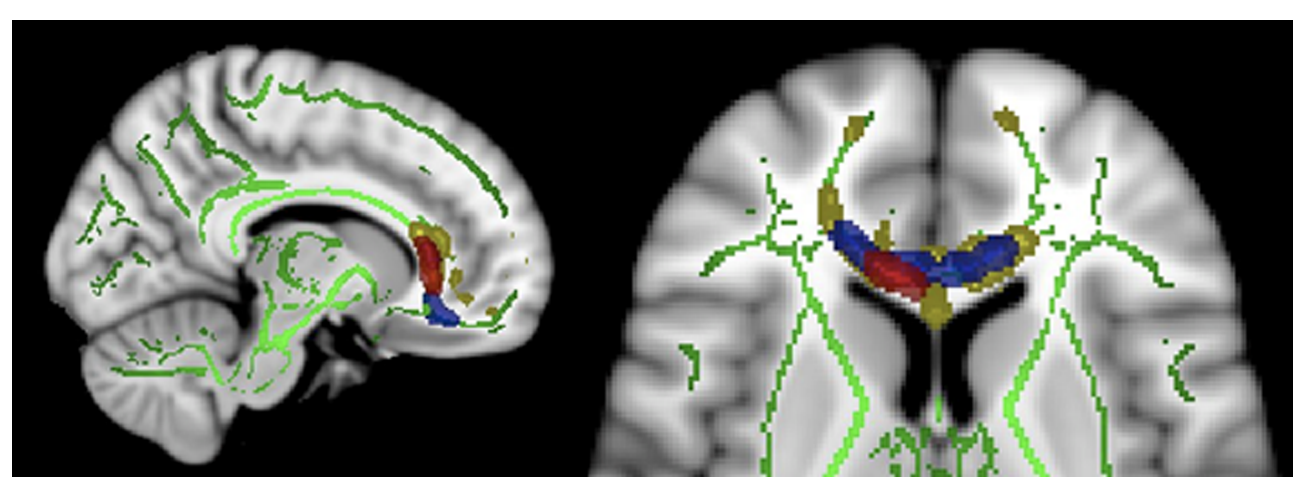

FIGURE 1 | Sagittal and axial views of Tract-Based Spatial Statistics results (MNI coordinates $\mathbf{m m}: \boldsymbol{x}=\mathbf{1 1}$ and $\boldsymbol{z}=\mathbf{7}$ respectively). Green: mean FA

(fractional anisotropy) skeleton. Yellow: higher correlation of FA values between monozygotic twin pairs compared to randomly generated pairs. Blue: reduced
FA values found in schizophrenia patients compared to controls reported in a previous study (Camchong et al., 2009). Red: reduced FA values found in relatives of SZ patients compared to controls in the current study. Images are radiologically oriented (left is right).
Table 1 | MNI (Montreal Neurological Institute) (Mazziotta et al., 1995) coordinates for regions showing high correlations between monozygotic twin pairs

\begin{tabular}{llll}
$\begin{array}{l}\text { John Hopkins University } \\
\text { white matter atlas }\end{array}$ & & $\begin{array}{l}\text { MNI } \\
\text { coordinates } \\
\text { (, } \boldsymbol{y , ~} \mathbf{z}\end{array}$ & $\begin{array}{l}\text { Volume - } \\
\text { microliters } \\
\text { (mm^3) }\end{array}$ \\
\hline Genu of corpus callosum & Left & $-12,33,5$ & 337 \\
& & $-1,25,5$ & 37 \\
Anterior cingulum & Right & $13,30,11$ & 324 \\
& Left & $-7,32,12$ & 120 \\
Forceps minor & Right & $14,45,6$ & 61 \\
& Left & $-15,54,-5$ & 55 \\
& & $-17,39,16$ & 54 \\
& Right & $13,34,17$ & 52 \\
& & $17,50,18$ & 52 \\
\hline
\end{tabular}

Table 2 | MNI (Montreal Neurological Institute) (Mazziotta et al., 1995) coordinates for regions showing reduced $F A$ values in relatives of schizophrenia patients when compared to controls.

\begin{tabular}{llll}
\hline John Hopkins University atlas & $\begin{array}{l}\text { MNI } \\
\text { coordinates } \\
\boldsymbol{x}, \boldsymbol{y}, \boldsymbol{z}\end{array}$ & $\begin{array}{l}\text { Volume - } \\
\text { microliters } \\
\text { (mm^3) }\end{array}$ \\
\hline Genu of corpus callosum & Right & $\begin{array}{l}8,28,7 \\
15,36,0\end{array}$ & $\begin{array}{c}137 \\
34\end{array}$ \\
\end{tabular}

connectivity previously found in schizophrenia patients (Sun et al., 2003; Fujiwara et al., 2007; Kubicki et al., 2008; Kyriakopoulos et al., 2008b; Camchong et al., 2009; Fitzsimmons et al., 2009).

To our knowledge, this is the first study that examined heritability of white matter integrity in MZ twin pairs. Our hypothesis proposing that MZ twins would have a stronger degree of correlation than in pairs of non-related individuals was supported, suggesting that white matter connectivity in frontal regions is moderately heritable, an important criterion in the development of an endophenotype. As shown in Figure 1, regions that have previously shown to have reduced white matter integrity in individuals with schizophrenia (Camchong et al., 2009) overlapped regions that were found to be highly correlated between MZ twin pairs, suggesting that schizophrenia-associated reduced white matter integrity in this region may be a heritable trait.

To further investigate the possibility that white matter disorganization in medial frontal regions is a schizophrenia-related endophenotype, white matter connectivity was examined in first-degree biological relatives of schizophrenia patients. Biological relatives of schizophrenia patients represent a sample of individuals at risk for developing the disease, without the confounding effects of the manifestation of the disease itself. Since present results showed that relatives of schizophrenia patients had frontal white matter connectivity abnormalities previously found in schizophrenia patients, these abnormalities might be related to the risk for developing the disease, and not to specific factors of the disease process itself such as symptom subtypes, illness duration, and/or medication effects. It should also be noted that regions previously found to have altered white matter integrity in schizophrenia patients (Camchong et al., 2009) are more widespread than in the relatives of schizophrenia patients (see Figure 1), suggesting that these white matter alterations are found in a lesser degree in individuals at risk for developing the disease than in patients.

Frontal white matter structures (genu of corpus callosum, anterior cingulum and forceps minor) found to have reduced FA in relatives in the present study connect lateral and medial surfaces of the frontal lobes and connect the left and right hemisphere through the corpus callosum. Evidence from studies on schizophrenia patients is consistent with altered brain connectivity in frontal regions found in the present study. In addition, previous studies have proposed a link between severity of symptomatology as well as cognition deficits and altered frontal brain connectivity (Manoach et al., 2007; Kubicki et al., 2008; Skelly et al., 2008; Takei et al., 2008; Camchong et al., 2009; Spoletini et al., 2009). Taken together, frontal regions that have shown altered white matter integrity in relatives of schizophrenia patients in the present study as well as in schizophrenia patients 
in previous studies (see Figure 1) (Sun et al., 2003; Fujiwara et al., 2007; Kubicki et al., 2008; Kyriakopoulos et al., 2008b; Camchong et al., 2009; Fitzsimmons et al., 2009), might be effective indicators of disease risk (endophenotype), because they manifest as a function of shared genes (not disease state).

There are four improvements related to research design applicable to this study. First, heritability of frontal white matter connectivity could be better addressed by examining groups with different degrees of shared genes. Future studies including dizygotic twins and siblings would add to the present findings by investigating parametric variations of genetic overlap. Similarly, a second caveat is that our sample representing individuals with a genetic liability to schizophrenia had potentially different degrees of relationship with the schizophrenia patients (parents, offspring and siblings). As a result, there is potentially greater heterogeneity of genetic liability in the present study as well as a large distribution of age in the relative sample. Third, the analysis of schizophrenia patients would have added to the results of the current study by allowing a direct comparison to relatives. The examination of relatives of schizophrenia patients, however, has the distinct advantage of not confounding the results with effects of antipsychotic medications and/or manifestation of the disease that schizophrenia patients would add. Fourth, the present study used a cross-sectional design with the relatives of schizophrenia patients. Future studies examining changes in frontal white matter integrity across time in individuals at risk for developing schizophrenia (tracking relatives who develop or don't develop the disease later on) would add to the schizophrenia literature.

In summary, the present study reports on the degree to which white matter disorganization in medial frontal regions is a schizophrenia-related endophenotype. The present study adds to the schizophrenia literature by providing preliminary evidence proposing frontal white matter integrity as a potential biological marker for schizophrenia based on Gottesman and Gould

\section{REFERENCES}

Basser, P. J., and Pierpaoli, C. (1996). Microstructural and physiological features of tissues elucidated by quantitative-diffusion-tensor MRI. J. Magn. Reson. B 111, 209-219.

Behrens, T. E., Woolrich, M. W., Jenkinson, M., Johansen-Berg, H., Nunes, R. G., Clare, S., Matthews, P. M., Brady, J. M. and Smith, S. M., (2003). Characterization and propagation of uncertainty in diffusion-weighted MR imaging. Magn. Reson. Med. 50, 1077-1088.

Camchong, J., MacDonald III, A., Bell, C., Mueller, B., and Lim, K. (2009). Altered functional and anatomical connectivity in schizophrenia. Schizophr. Bull. in press.

Cheung, V., Cheung, C., McAlonan, G. M., Deng, Y., Wong, J. G., Yip, L., Tai, K. S., Khong, P. L., Sham, P. and Chua, S. E., (2008). A diffusion tensor imaging study of structural dysconnectivity in never-medicated, firstepisode schizophrenia. Psychol. Med. 38, 877-885.
Ellison-Wright, I., and Bullmore, E. (2009). Meta-analysis of diffusion tensor imaging studies in schizophrenia. Schizophr. Res. 108, 3-10.

Fitzsimmons, J., Kubicki, M., Smith, K., Bushell, G., Estepar, R. S., Westin, C. F., Nestor, P. G., Niznikiewicz, M. A., Kikinis, R., McCarley, R. W., and Shenton, M.E. (2009). Diffusion tractography of the fornix in schizophrenia. Schizophr. Res. 107, 39-46.

Fujiwara, H., Namiki, C., Hirao, K., Miyata, J., Shimizu, M., Fukuyama, H., Sawamoto, N., Hayashi, T., and Murai, T. (2007). Anterior and posterior cingulum abnormalities and their association with psychopathology in schizophrenia: a diffusion tensor imaging study. Schizophr. Res. 95, 215-222.

Gottesman, I. I., and Gould, T. D. (2003). The endophenotype concept in psychiatry: etymology and strategic intentions. Am. J. Psychiatry 160, 636-645.

Hoptman, M. J., Ardekani, B. A., Butler, P. D., Nierenberg, J., Javitt, D. C., and

endophenotype criteria (Gottesman and Gould, 2003). Previous studies have shown that altered frontal white matter is associated with schizophrenia (Sun et al., 2003; Fujiwara et al., 2007; Kubicki et al., 2008; Kyriakopoulos et al., 2008b; Camchong et al., 2009; Fitzsimmons et al., 2009). Second, frontal white matter integrity showed to be heritable within the MZ twin sample. Third, altered frontal white matter integrity was found in nonaffected family members at a higher rate than in the general population. It seems, therefore, that reduced white matter integrity in frontal regions of the brain is a heritable trait that appears to be associated with the genetic liability to schizophrenia.

Understanding brain abnormalities, such as frontal white matter connectivity alterations examined in the present study, aids in identifying traits that suggest genetic liability to schizophrenia because these physiological alterations are more intrinsically related to genetic alterations in schizophrenia patients than symptoms and cognition. Measures of frontal connectivity (such as fractional anisotropy measures) may be used in quantitative trait loci analyses in the search for schizophrenia genes. Future studies combining neuroimaging methods, neuropsychological assessment and molecular genetic assessment on participants with different degrees of genetic susceptibility to schizophrenia (patients, first and second degree relatives) will have a maximized power for identifying schizophrenia genes.

\section{ACKNOWLEDGMENTS}

This research was supported by the National Institute of Mental Health (R24MH069675, MH066629, MH079262 and MH060662), Department of Veterans Affairs Clinical Science Research and Development Program (Merit Review) to Scott R. Sponheim, Training Grant 5T32MH017069-26 for Jazmin Camchong, and the Center for Magnetic Resonance Research (BTRR - P41 RR008079 and NCC grant P30 NS057091). The authors would like to thank the subjects who participated in this study.

Lim, K. O. (2004). DTI and impulsivity in schizophrenia: a first voxelwise correlational analysis. Neuroreport 15 , 2467-2470.

Hoptman, M. J., Nierenberg, J., Bertisch, H. C., Catalano, D., Ardekani, B. A., Branch, C. A., and Delisi, L. E. (2008). A DTI study of white matter microstructure in individuals at high genetic risk for schizophrenia. Schizophr. Res. 106, 115-124.

Howell, D., (2001). Randomization Tests on Correlation Coefficients. available at: http://www.uvm.edu/ dhowell/ StatPages/Resampling/RandomCorr/ randomization_Correlation.html.

Howell, D., (2007). Randomization Tests. available at: http://www.uvm.edu/ $\sim$ dhowell/StatPages/Resampling/.

Kanaan, R.A., Kim, J.S., Kaufmann, W. E., Pearlson, G. D., Barker, G. J., and McGuire, P. K. (2005). Diffusion tensor imaging in schizophrenia. Biol. Psychiatry 58, 921-929.

Karlsgodt, K. H., Niendam, T.A., Bearden, C.E., and Cannon, T. D. (2009). White matter integrity and prediction of social and role functioning in subjects at ultra-high risk for psychosis. Biol Psychiatry 66, 562-569.

Kubicki, M., Park, H., Westin, C. F., Nestor, P. G., Mulkern, R. V., Maier, S. E., Niznikiewicz, M., Connor, E. E., Levitt, J. J., Frumin, M., Kikinis, R., Jolesz, F. A., McCarley, R.W., and Shenton, M.E. (2005).DTI and MTR abnormalities in schizophrenia: analysis of white matter integrity. Neuroimage 26, 1109-1118.

Kubicki,M.,Styner,M., Bouix, S., Gerig, G., Markant, D., Smith, K., Kikinis, R., McCarley, R. W., and Shenton, M. E. (2008). Reduced interhemispheric connectivity in schizophrenia-tractography based segmentation of the corpus callosum. Schizophr. Res. 106, 125-131.

Kubicki, M., Westin, C. F., Nestor, P. G., Wible, C. G., Frumin, M., Maier, S. E., Kikinis, R.,Jolesz,F.A., McCarley, R. W., and Shenton, M. E. (2003). Cingulate fasciculus integrity disruption in schizophrenia: a magnetic resonance diffusion tensor imaging study. Biol. Psychiatry 54, 1171-1180. 
Kyriakopoulos, M., Bargiotas, T., Barker, G. J., and Frangou, S. (2008a). Diffusion tensor imaging in schizophrenia. Eur. Psychiatry 23, 255-273.

Kyriakopoulos, M., Vyas, N. S., Barker, G. J., Chitnis, X. A., and Frangou, S. (2008b). A diffusion tensor imaging study of white matter in early-onset schizophrenia. Biol. Psychiatry 63, 519-523.

Manoach, D. S., Ketwaroo, G. A., Polli, F. E., Thakkar, K. N., Barton, J. J., Goff, D. C., Fischl, B., Vangel, M., and Tuch, D. S. (2007). Reduced microstructural integrity of the white matter underlying anterior cingulate cortex is associated with increased saccadic latency in schizophrenia. Neuroimage 37, 599-610.

Mazziotta, J. C., Toga, A. W., Evans, A., Fox, P., and Lancaster, J. (1995). A probabilistic atlas of the human brain: theory and rationale for its development. The International Consortium for Brain Mapping (ICBM). Neuroimage 2, 89-101.

Munoz Maniega, S., Lymer, G. K., Bastin, M.E., Marjoram, D., Job, D. E., Moorhead, T. W., Owens, D. G., Johnstone, E. C., McIntosh, A. M., and Lawrie, S. M. (2008). A diffusion tensor MRI study of white matter integrity in subjects at high genetic risk of schizophrenia. Schizophr. Res. 106, 132-139.

Nichols, T. E., and Holmes, A. P. (2002). Nonparametric permutation tests for functional neuroimaging: a primer with examples. Hum. Brain Mapp. 15, 1-25.

Reese, T. G., Heid, O., Weisskoff, R. M. and Wedeen, V. J. (2003). Reduction of eddy-current-induced distortion in diffusion MRI using a twice-refocused spin echo. Magn. Reson. Med. 49, 177-182.

Rueckert, D., Sonoda, L. I., Hayes, C., Hill, D. L., Leach, M. O., and Hawkes, D. J. (1999). Nonrigid registration using free-form deformations: application to breast MR images. IEEE Trans. Med. Imaging 18, 712-721.

Schlosser, R. G., Nenadic, I., Wagner, G., Gullmar, D., von Consbruch, K. Kohler, S., Schultz, C. C., Koch, K., Fitzek, C., Matthews, P. M., Reichenbach, J. R., and Sauer, H. (2007). White matter abnormalities and brain activation in schizophrenia: a combined DTI and fMRI study. Schizophr. Res. 89, 1-11.

Skelly, L. R., Calhoun, V., Meda, S. A., Kim, J., Mathalon, D. H., and Pearlson, G. D. (2008). Diffusion tensor imaging in schizophrenia: relationship to symptoms. Schizophr. Res. 98, 157-162.

Smith, S. M., Jenkinson, M., JohansenBerg, H., Rueckert, D., Nichols, T. E., Mackay, C. E., Watkins, K. E., Ciccarelli, O., Cader, M.Z., Matthews, P. M., and Behrens, T. E. (2006). Tract-based spatial statistics: voxelwise analysis of multi-subject diffusion data. Neuroimage 31, 1487-1505.
Smith, S. M., Jenkinson, M., Woolrich, M. W., Beckmann, C. F., Behrens, T. E., Johansen-Berg, H., Bannister, P. R., De Luca, M., Drobnjak, I., Flitney, D. E., Niazy, R. K., Saunders, J., Vickers, J., Zhang, Y., De Stefano, N., Brady, J. M., and Matthews, P.M. (2004). Advances in functional and structural MR image analysis and implementation as FSL. Neuroimage 23(Suppl. 1), S208-S219.

Spoletini, I., Cherubini, A., Di Paola, M., Banfi, G., Rusch, N., Martinotti, G., Bria, P., Rubino, I. A., Siracusano, A., Caltagirone, C., and Spalletta, G. (2009). Reduced fronto-temporal connectivity is associated with frontal gray matter density reduction and neuropsychological deficit in schizophrenia. Schizophr. Res. 108, 57-68.

Sun, Z., Wang, F., Cui, L., Breeze, J., Du, X. Wang, X., Cong, Z., Zhang, H., Li, B., Hong, N., and Zhang, D. (2003). Abnormal anterior cingulum in patients with schizophrenia: a diffusion tensor imaging study. Neuroreport 14, 1833-1836.

Takei, K., Yamasue, H., Abe, O., Yamada, H., Inoue, H., Suga, M., Sekita, K., Sasaki, H., Rogers, M., Aoki, S., and Kasai, K. (2008). Disrupted integrity of the fornix is associated with impaired memory organization in schizophrenia. Schizophr. Res. 103, 52-61.

Whitfield-Gabrieli, S., Thermenos, H. W., Milanovic, S., Tsuang, M.
T., Faraone, S. V., McCarley, R. W., Shenton, M. E., Green, A. I., NietoCastanon, A., LaViolette, P., Wojcik, J., Gabrieli, J. D., and Seidman, L. J. (2009). Hyperactivity and hyperconnectivity of the default network in schizophrenia and in first-degree relatives of persons with schizophrenia. Proc. Natl. Acad. Sci. U.S.A. 106, 1279-1284.

Conflict of Interest Statement: The authors declare that the research was conducted in the absence of any commercial or financial relationships that could be construed as a potential conflict of interest.

Received: 02 June 2009; paper pending published: 08 July 2009; accepted: 04 October 2009; published online: 26 October 2009.

Citation: Camchong J, Lim KO, Sponheim SR and MacDonald III AW (2009) Frontal white matter integrity as an endophenotype for schizophrenia: diffusion tensor imaging in monozygotic twins and patients' nonpsychotic relatives. Front. Hum. Neurosci. 3:35. doi: 10.3389/neuro.09.035.2009

Copyright (c) 2009 Camchong, Lim, and MacDonald III. This is an open-access article subject to an exclusive license agreement between the authors and the Frontiers Research Foundation, which permits unrestricted use, distribution, and reproduction in any medium, provided the original authors and source are credited. 